DOI : $10.24850 / \mathrm{j}-$ tyca-2022-01-07

Artículos

\title{
Estudio hidrológico de una cuenca para la evaluación del potencial de una pico central hidroeléctrica en una zona rural
}

\section{Hydrological study of a basin for the evaluation of the potential of a pico hydroelectric power station in a rural area}

Inés Aberlí Nieves-Vilchez ${ }^{1}$, ORCID: 0000-0001-6673-5331

Hiram Joshua Castillo-Infante2, ORCID: 0000-0001-8206-6393

Josué Isac Carillo-Espinoza³, ORCID: 0000-0002-4241-2834

Noé Benjamín Pampa-Quispe ${ }^{4}$, ORCID: 0000-0001-8072-249X

${ }^{1}$ Escuela Profesional de Ingeniería Ambiental, Universidad Peruana Unión, Lima, Perú, inesnieves@upeu.edu.pe

²Escuela Profesional de Ingeniería Ambiental, Universidad Peruana Unión, Lima, Perú, hiramcastillo@upeu.edu.pe

${ }^{3}$ Facultad de Ingeniería y Arquitectura, Universidad Peruana Unión, Lima, Perú, josuecarrilloe@upeu.edu.pe 
${ }^{4}$ Facultad de Ingeniería y Arquitectura, Universidad Peruana Unión, Lima, Perú/Departamento de Ingeniería, Universidad Nacional de Barranca, Barranca, Perú, noepampa@upeu.edu.pe

Autor para correspondencia: Noé Benjamín Pampa-Quispe, noepampa@upeu.edu.pe

\section{Resumen}

El objetivo del estudio fue estimar los escurrimientos promedio mensuales de una cuenca para el desarrollo de una pico central hidroeléctrica en un área rural-montañosa de la selva (Satipo) del Perú. Debido a la ausencia de datos hidrometeorológicos en cuencas montañosas y remotas en el Perú, una estimación hidrológica resulta indispensable para determinar el potencial hidroenergético en la cuenca de estudio. Primero se determinaron los parámetros geomorfológicos (de forma y de relieve) de la cuenca $y$, posteriormente, se realizó un análisis de precipitación tomando en cuenta los datos de 17 estaciones meteorológicas. Para verificar la homogeneidad de los registros pluviométricos, se realizó un análisis de consistencia mediante un análisis de dobles acumulaciones. Para estimar los caudales de la cuenca de estudio, a falta de información hidrométrica, se utilizó el método de transposición de caudal, en el cual se transportaron los caudales de la cuenca Ourohuari. Asimismo, se comprobó que las características geomorfológicas e hidrometeorológicas resultaran similares, para ello se realizó una prueba $t$-test para muestras independientes, con el fin de verificar la similitud en la precipitación anual 
entre ambas cuencas. Finalmente, se puede concluir, las características geomorfológicas y el caudal regular durante todo el año en la cuenca Cashingari favorece el desarrollo de una pico central hidroeléctrica.

Palabras clave: caudal, precipitación, evaluación hidrológica, pico central.

\section{Abstract}

The goal of the study was to estimate the average monthly runoff from a basin for the development of a pico hydropower in a rural-mountainous area of the jungle (Satipo) on Peru. Due to the lack of hydrometeorological data in hilly and remote basins in Peru, a hydrological estimate is essential to determine the hydroenergy potential in the study basin. First, the geomorphological parameters (shape and relief parameters) of the basin were determined and, subsequently, a precipitation analysis was made considering the data from 17 meteorological stations. To verify the homogeneity of the rainfall records, a consistency analysis was carried out using a double accumulation analysis. To estimate the flows of the study basin, in the absence of hydrometric information, the flow transposition method was used, in which the flows of the Ourohuari basin were transported. Likewise, it was verified that the geomorphological and hydrometeorological characteristics are similar, for this, a $t$-test was carried out for independent samples to verify the similarity in the annual precipitation between both basins. Finally, it can be concluded that the 
geomorphological characteristics and flow regular throughout the year in the Cashingari basin are favoring the development of a pico hydropower.

Keywords: Flow, precipitation, hydrological assessment, pico hydroelectric power.

Recibido: $31 / 12 / 2018$

Aceptado: $25 / 01 / 2021$

\section{Introducción}

La energía hidroeléctrica es una de las fuentes renovables más importantes para la producción de electricidad, pues es limpia y confiable. Este tipo de energía deriva de la caída del agua tanto de los ríos como de los arroyos que fluyen cuesta abajo a lo largo del curso del río debido a la fuerza de la gravedad; la energía asociada con el agua que fluye se conoce como energía cinética (Kusre, Baruah, Bordoloi, \& Patra, 2010). Es por ello que la cantidad de hidroenergía generada está en función de la altura y el caudal de agua (Soulis, Manolakos, Anagnostopoulos, \& Papantonis, 2016). 
La mayoría de los estudios previos que evaluaron la generación potencial de energía mediante centrales hidroeléctricas a pequeña escala se enfocaron en regiones montañosas debido a que presentan una geografía empinada y los ríos proporcionan alturas naturales (Fujii et al., 2017).

Las pico centrales hidroeléctricas que hacen referencia a las instalaciones con niveles de generación menores a 5 kW (Chouhan, Kisheorey, \& Shah, 2017) normalmente es un sistema que se implementa en áreas rurales o montañosas (Uniyal, Kanojia, \& Pandey, 2016); es la tecnología más rentable y confiable para producir electricidad fuera de la red (Edeoja, Ibrahim, \& Kucha, 2015).

Para un proyecto hidroeléctrico a pequeña escala, los datos sobre los procesos hidrológicos son fundamentales tanto en el diseño como en la operación (Soulis et al., 2016), pues tales datos se necesitan para la comprensión del comportamiento y características hidrológicas de una cuenca (Ezemonye \& Emeribe, 2013); además, la evaluación precisa y confiable de los datos hidrológicos conduce a una planificación exitosa (Sidek et al., 2013). Sin embargo, los estudios han demostrado que la recopilación de información precisa y consistente para la mayoría de las cuencas en los países en desarrollo es deficiente o inadecuada cuando están disponibles, lo cual representa un gran desafío para los servicios hidrológicos de cada país (Negrel, Kosuth, \& Bercher, 2011). Este problema puede atribuirse a la ausencia general de estaciones de medición (Ezemonye \& Emeribe, 2013) y también al coste que implica la generación de datos de descarga (Soulis et al., 2016). 
La ausencia de datos hidrológicos o información inexacta conduce a una falta de motivación para el desarrollo de la energía hidroeléctrica en países en desarrollo (Kusre et al., 2010).

El caso del Perú no es la excepción, pues a pesar de estar entre los 20 países con mayor oferta hídrica en el mundo, que asciende a 77534 $\mathrm{m}^{3}$ /hab/año (Jimenez \& Galizia, 2012), uno de los principales inconvenientes para la puesta en marcha de proyectos hidroenergéticos es la ausencia de información hidrometeorológica, que principalmente sucede en cuencas de zonas rurales.

Además, el Perú, a pesar de que es favorecido por la disponibilidad de fuentes de energía autóctonas, incluyendo de manera principal la energía hidráulica (Meier, Zolezzi, Bogach, Muir, \& Bazex, 2011), aún tiene brechas de desigualdad con respecto al acceso al servicio de electricidad. Del área urbana, un $98.9 \%$ tiene acceso y sólo un $74.5 \%$ cuenta con servicio en el área rural; además, en la región selva, donde es más evidente la desigualdad, el área urbana tiene un $97.8 \%$ de acceso a la electricidad frente al $55.7 \%$ del área rural (Figura 1); esto presenta un gran desafío de electrificación rural para dicha zona del país (World Bank, 2017). 
Tecnología y

Ciencias $₫$ Agua
2022, Instituto Mexicano de Tecnología del Agua

Open Access bajo la licencia CC BY-NC-SA 4.0

(https://creativecommons.org/licenses/by-nc-sa/4.0/)



Figura 1. Acceso a la electrificación en el Perú (World Bank, 2017).

Por este antecedente, el objetivo del trabajo es realizar un estudio hidrológico de una microcuenca para el desarrollo de una pico central hidroeléctrica en una zona rural-montañosa de la selva central del Perú, con el fin de brindar energía eléctrica.

\section{Materiales y métodos}




\section{Parámetros geomorfológicos}

Los parámetros geomorfológicos desempeñan un papel muy importante en el estudio del comportamiento hidrológico de las cuencas hidrográficas (Gavit, Purohit, Singh, Kothari, \& Jain, 2016). Para ello, se determinaron los parámetros generales, parámetros de forma y parámetros de relieve.

Parámetros generales. A través del Sistema de Información Geográfica (SIG), mediante el software ArcGis (v. 10.2), se determinaron las características generales de la microcuenca Cashingari, entre las que se encuentran área, perímetro y longitud del cauce principal.

Parámetros de forma. Los parámetros de forma influyen en el tiempo necesario para que el agua de la parte remota de la cuenca llegue a la salida de la misma (Bilewu, Sule, \& Ayanshola, 2015), para ello se mide cuantitativamente a través de varios factores, como coeficiente de compacidad, factor de forma, índice de elongación, índice de circularidad y rectángulo equivalente; las ecuaciones utilizadas para cada parámetro de muestran en Ia Tabla 1.

Tabla 1. Parámetros de forma.

\begin{tabular}{|l|l|l|}
\hline Parámetros & Ecuaciones & Nomenclatura \\
\hline
\end{tabular}




\begin{tabular}{|l|c|l|}
\hline $\begin{array}{l}\text { Coeficiente } \\
\text { de } \\
\text { compacidad }\end{array}$ & $C c=0.282 \times \frac{P}{\sqrt{A}}$ & $\begin{array}{l}P=\text { perímetro de la cuenca (km) } \\
A=\text { área de la cuenca }\left(\mathrm{km}^{2}\right)\end{array}$ \\
\hline $\begin{array}{l}\text { Factor de } \\
\text { forma }\end{array}$ & $F F=\frac{A}{L^{2}}$ & $\begin{array}{l}A=\text { área de la cuenca } \\
L=\text { longitud del cauce principal }\end{array}$ \\
\hline $\begin{array}{l}\text { Índice de } \\
\text { elongación }\end{array}$ & $R_{e}=1.129 \frac{\sqrt{A}}{L}$ & $\begin{array}{l}A=\text { área de la cuenca } \\
L=\text { longitud de la cuenca }\end{array}$ \\
\hline $\begin{array}{l}\text { Índice de } \\
\text { circularidad }\end{array}$ & $R_{c}=\frac{4 \pi A}{P^{2}}$ & $\begin{array}{l}A=\text { área de la cuenca } \\
P=\text { perímetro de la cuenca }\end{array}$ \\
\hline $\begin{array}{l}\text { Rectángulo } \\
\text { equivalente }\end{array}$ & $\begin{array}{l}L, l \\
\frac{K c}{1.128}[1\end{array}$ & $\begin{array}{l}L=\text { longitud del lado mayor del } \\
\text { rectángulo } \\
I=\text { longitud del lado menor del } \\
\text { rectángulo } \\
K C=\text { coeficiente de compacidad } \\
A=\text { área de la curva }\end{array}$ \\
\hline
\end{tabular}

Parámetros de relieve. Algunos parámetros de relieve se determinaron a través del software ArcGis (v. 10.2), como desnivel altitudinal, cota mínima, cota máxima, elevación media, coordenada del centroide $X$, coordenada del centroide $Y$. La pendiente de la cuenca se determinó a través de la ecuación mostrada en la Tabla 2.

Tabla 2. Parámetros de relieve. 


\begin{tabular}{|c|c|l|}
\hline Parámetro & Ecuación & \multicolumn{1}{|c|}{ Leyenda } \\
\hline $\begin{array}{c}\text { Pendiente } \\
\text { de la } \\
\text { cuenca } \\
\text { (Sc) }\end{array}$ & $S c=\frac{D}{A} x \sum_{i=n}^{n=1} L i$ & $\begin{array}{l}D=\text { equidistancia o desnivel } \\
\text { constante entre curas de nivel }(\mathrm{km}) \\
\end{array}$ \\
& & $\begin{array}{l}A=\text { área de la cuenca }\left(\mathrm{km}^{2}\right) \\
L_{i}=\text { longitud de la cura de nivel "i" } \\
(\mathrm{km})\end{array}$ \\
\hline
\end{tabular}

\section{Precipitación}

Red de estaciones. Para la presente investigación se tomaron en cuenta 17 estaciones pluviométricas (Figura 2): Comas, Huasahuasi, Ingenio, Jauja, La Oroya, Mazamari, Mezapata, Oxapampa, Pampa Whaley, Pichanaki, Puerto Ocopa, Ricran, Runatullo, San Miguel de Bocaz, Santa Ana, Satipo y Tarma, las cuales tienen las siguientes características:

- La información histórica de precipitación total mensual y total anual de las estaciones está registrada por el Servicio Nacional de Meteorología Hidrología (SENAMHI).

- De las 17 estaciones elegidas, 11 pertenecen a la cuenca del río Perené; cuatro, a la cuenca del río Mantaro, y dos a la cuenca del río Pachitea. 
Tecnología y

Ciencias Agua
2022, Instituto Mexicano de Tecnología del Agua

Open Access bajo la licencia CC BY-NC-SA 4.0

(https://creativecommons.org/licenses/by-nc-sa/4.0/)

- Las estaciones Huasahuasi, Tarma, Ricran, Comas y Runatullo se ubican en la parte alta de la cuenca del río Perené; las estaciones Mezapata, Pampa Whaley y Pichanaki en la cuenca media; y las estaciones Satipo, Mazamari y Puerto Ocopa en la parte baja de la cuenca.



Figura 2. Ubicación de las estaciones pluviométricas. 
Tecnología y

Ciencias $₫$ Agua
2022, Instituto Mexicano de Tecnología del Agua

Open Access bajo la licencia CC BY-NC-SA 4.0

(https://creativecommons.org/licenses/by-nc-sa/4.0/)

Las estaciones de donde se obtuvieron los datos pluviométricos, el periodo de registro y los años de registro se muestran en la Tabla 3.

Tabla 3. Estaciones de registro.

\begin{tabular}{|c|c|c|}
\hline Estación & Periodo & $\begin{array}{l}\text { Años de } \\
\text { registro }\end{array}$ \\
\hline Comas & $1995-2010$ & 16 \\
\hline Tarma & $1996-2010$ & 15 \\
\hline Ronatullo & $1993-2010$ & 18 \\
\hline Huasahuasi & $1996-2009$ & 14 \\
\hline Pichanaki & $2001-2010$ & 10 \\
\hline Satipo & $2001-2012$ & 12 \\
\hline $\begin{array}{l}\text { Puerto } \\
\text { Ocopa }\end{array}$ & $1964-1978,1980-1982,1999-2010$ & 30 \\
\hline Oxapampa & $2001-2010$ & 10 \\
\hline $\begin{array}{l}\text { San miguel } \\
\text { de Bocaz }\end{array}$ & $1964-1973$ & 10 \\
\hline $\begin{array}{l}\text { Pampa } \\
\text { Whaley }\end{array}$ & 1964-1969, 1971-1972 у 1977 & 9 \\
\hline Mezapata & 1991-1997 y 1999-2003 & 12 \\
\hline Mazamari & $1969,1976-1977$ у $1979-1980$ & 5 \\
\hline Santa Ana & $2000-2010$ & 11 \\
\hline
\end{tabular}


Tecnología y

Ciencias $₫$ Agua
2022, Instituto Mexicano de Tecnología del Agua

Open Access bajo la licencia CC BY-NC-SA 4.0

(https://creativecommons.org/licenses/by-nc-sa/4.0/)

\begin{tabular}{|l|c|c|}
\hline Ricran & $2000-2010$ & 11 \\
\hline La Oroya & $2000-2010$ & 11 \\
\hline Jauja & $2000-2010$ & 11 \\
\hline Ingenio & $2000-2010$ & 11 \\
\hline
\end{tabular}

Análisis de consistencia de precipitación. Para el análisis de consistencia de los registros de precipitación se utilizó el método de análisis de dobles acumulaciones, muy usado para verificar la homogeneidad de registros pluviométricos; el fin de este análisis es detectar alguna irregularidad en las estaciones durante el tiempo de registro.

Para efectos del análisis de consistencia de las 17 estaciones, se formaron cuatro grupos, teniendo en cuenta la ubicación espacial (altitud) y el periodo de registro, como se muestra en la Tabla 4.

Tabla 4. Grupos formados para el análisis de consistencia.

\begin{tabular}{|l|c|c|}
\hline Grupo & Estaciones & Periodo de registro \\
\hline Grupo 1 & $\begin{array}{c}\text { Comas, Ronatullo, Santa Ana, } \\
\text { Jauja e Ingenio }\end{array}$ & $2000-2010$ \\
\hline Grupo 2 & $\begin{array}{c}\text { Tarma, Huasahuasi, Ricran y La } \\
\text { Oroya }\end{array}$ & $2000-2009$ \\
\hline Grupo 3 & $\begin{array}{c}\text { Pichanaki, Satipo, Puerto Ocopa } \\
\text { y Oxapampa }\end{array}$ & $2001-2010$ \\
\hline
\end{tabular}




\begin{tabular}{|l|c|c|}
\hline Grupo 4 & $\begin{array}{c}\text { San Miguel de Bocaz y Pampa } \\
\text { Whaley }\end{array}$ & $1964-1969$ \\
\hline
\end{tabular}

Las estaciones Mazamari y Mezapata no se tomaron en cuenta debido a que sus periodos de registro no presentan años en común.

Precipitación de la cuenca. Para determinar la precipitación aereal de la microcuenca Cashingari se utilizó el método de las isoyetas debido a que es el de mejor aproximación y representatividad al ingresar los efectos orográficos de la cuenca.

Para la construcción de las isoyetas a nivel total anual y medias mensuales se emplearon las 17 estaciones pluviométricas: Comas, Huasahuasi, Ingenio, Jauja, La Oroya, Mazamari, Mezapata, Oxapampa, Pampa Whaley, Pichanaki, Puerto ocopa, Ricran, Runatullo, San Miguel de Bocaz, Santa Ana, Satipo y Tarma.

Para determinar la precipitación total anual de la microcuenca se realizó la distribución de isoyetas cada $50 \mathrm{~mm}$ y para la precipitación mensual cada $10 \mathrm{~mm}$. Aplicando la ecuación que se presenta a continuación, se obtiene la precipitación aereal:

$$
P=\frac{\sum_{i=1}^{n}\left[\frac{P_{i}+P_{i+1}}{2}\right] x A_{i}}{\sum_{i=1}^{n} A_{i}}
$$

donde:

$\mathrm{P}_{\mathrm{i}}=$ valor de precipitación de la isoyeta $i$. 
$A_{i}=$ área incluida entre dos isoyetas consecutivas $(i$ y $(i+1)$. $\mathrm{n}=$ número de isoyetas.

\section{Caudal promedio}

Debido a la ausencia de información hidrométrica dentro de la cuenca Cashingari se determinaron los caudales promedios mensuales a través del método de regionalización de área de drenaje (transposición de caudales), que consiste en transferir los datos de caudal de una cuenca cercana con la cuenca de estudio (Fatoyinbo, Stretch, Amoo, \& Allopi, 2017). Este método es más confiable cuando la proporción del área de drenaje entre ambas cuencas está dentro del rango de 0.5-1.5 (Fry, Hunter, Phanikumar, Fortin, \& Gronewold, 2013).

Para determinar el caudal se deben conocer las áreas de la cuenca de estudio y la cuenca que tiene similares características; además, la precisión en la estimación del caudal dependerá de la proximidad y similitudes en el área de la cuenca, y demás características físicas y climáticas (Hortness, 2006).

Las condiciones similares que tienen que presentar ambas cuencas son hidrometeorológicas (precipitación, temperatura, humedad); para ello, con el fin de determinar que la precipitación es similar se realizó una 
prueba $t$-student para muestras independientes a un nivel de confianza del $95 \%$. Asimismo, las características geomorfológicas (área, perímetro, elevación, pendiente, demás parámetros y coeficientes, etc.) deben ser similares. La ecuación para transponer los datos de caudal de una cuenca a otra se presenta a continuación:

$Q_{s}=Q_{c} \times\left(\frac{A_{s}}{A_{c}}\right)$

donde:

$Q_{s}=$ caudal de la cuenca sin información.

$Q_{c}=$ caudal de la cuenca con información.

$A_{s}=$ área de la cuenca sin información.

$A_{c}=$ área de la cuenca con información.

\section{Máximas avenidas}

Caudales de avenidas de la cuenca. Para determinar los caudales de avenida de la microcuenca Cashingari se ha utilizado la ecuación envolvente de Creager, que para el caso específico del Perú ha sido adaptada por Wolfang Trau y Raúl Gutierrez (Trau \& Gutierrez, 1979) para 
diferentes periodos de retorno, según las regiones y cuencas que integran. La ecuación de Creager es una de las más usadas y conocidas debido a que proporciona una estimación de la descarga máxima esperada en función del área de drenaje (De-Lima, Marcellini, Neill, \& Salla, 2017):

$Q_{\text {máx }}=\left(C_{1}+C_{2}\right) \times \log (T) \times A^{m \times A^{-n}}$

donde:

$Q_{\text {máx }}=$ caudal $\left(\frac{\mathrm{m}^{3}}{\mathrm{~s}}\right)$.

$C_{1}=$ coeficiente de escala, adimensional.

$C_{2}=$ coeficiente de escala, adimensional.

$T=$ período de retorno (año).

$A=$ área de la cuenca $\left(\mathrm{km}^{2}\right)$.

$m=$ exponente, adimensional.

$n=$ exponente adimensional.

De la ecuación descrita, los exponentes $m$ y $n$ determinan la forma y la pendiente de la curva, y los coeficientes $C_{1}$ y $C_{2}$ la escala de la curva. Los valores de los exponentes y coeficientes mencionados se muestran en la Tabla 5. 
Tecnología y

Ciencias $₫$ Agua
2022, Instituto Mexicano de Tecnología del Agua

Open Access bajo la licencia CC BY-NC-SA 4.0

(https://creativecommons.org/licenses/by-nc-sa/4.0/)

Tabla 5. Coeficientes y exponentes según región del Perú (Trau \& Gutierrez, 1979).

\begin{tabular}{|c|c|c|c|c|c|}
\hline Región & Cuencas & C1 & C2 & m & n \\
\hline $\begin{array}{c}\text { Costa norte } \\
\text { (frontera) }\end{array}$ & Tumbes a Piura & 1.01 & 4.37 & 1.02 & 0.04 \\
\hline Costa norte & Cascajal a Santa & 0.10 & 1.28 & 1.02 & 0.04 \\
\hline Sierra norte & Alto Marañón & 0.27 & 1.48 & 1.02 & 0.04 \\
\hline $\begin{array}{c}\text { Costa } \\
\text { central }\end{array}$ & $\begin{array}{c}\text { Lacramarca a Camaná- } \\
\text { Majes }\end{array}$ & 0.09 & 0.36 & 1.24 & 0.04 \\
\hline Costa sur & Quilca a Caplina & 0.11 & 0.26 & 1.24 & 0.04 \\
\hline Sierra & $\begin{array}{c}\text { Mantaro, Apurímac y } \\
\text { Urubamba }\end{array}$ & 0.18 & 0.31 & 1.24 & 0.04 \\
\hline Selva & $\begin{array}{c}\text { Ucayali, Bajo Marañón, } \\
\text { Madre de Dios y } \\
\text { Amazonas }\end{array}$ & 0.22 & 0.37 & 1.24 & 0.04 \\
\hline
\end{tabular}

\section{Resultados}

\section{Descripción de la cuenca}


La microcuenca Cashingari está (Figura 3) dentro de la subcuenca Sanibeni y de la cuenca Satipo; como cuenca general se encuentra la cuenca del río Perené. El área de la microcuenca es de $6.82 \mathrm{~km}^{2}$ y su longitud de 14.7 km; tiene como máxima elevación 1593 msnm y como elevación mínima 597 msnm. La microcuenca Cashingari está clasificada como una cuenca muy pequeña debido a que su área es menor a los 25 $\mathrm{km}^{2}$. 
Tecnología y

Ciencias $\stackrel{\Xi}{\approx}$ gua
2022, Instituto Mexicano de Tecnología del Agua

Open Access bajo la licencia CCBY-NC-SA 4.0

(https://creativecommons.org/licenses/by-nc-sa/4.0/)



Figura 3. Ubicación de cuenca de estudio.

\section{Parámetros geomorfológicos de la cuenca}

La Tabla 6 muestra los parámetros geomorfológicos de la cuenca. Se pueden apreciar los parámetros de forma: coeficiente de compacidad 
Tecnología y

Ciencias $₫$ Agua
2022, Instituto Mexicano de Tecnología del Agua

Open Access bajo la licencia CC BY-NC-SA 4.0

(https://creativecommons.org/licenses/by-nc-sa/4.0/)

(1.89), índice de elongación (0.20) y factor de forma (0.44). Esto indica una figura alargada de la cuenca cuando el factor de forma es inferior a 1; existe una baja probabilidad de ocurrencia de avenidas (Reyes, Barroso, \& Carvajal, 2010). Por otro lado, los parámetros de relieve muestran que la elevación media de la cuenca es de 1144 msnm; la pendiente mediante el método de Alvord es del $42 \%$, y la pendiente del cauce principal es del $22 \%$.

Tabla 6. Características geomorfológicas de la microcuenca Cashingari.

\begin{tabular}{|c|c|c|}
\hline Tipo de & \multicolumn{2}{|c|}{ Parámetro } \\
\hline \multirow{3}{*}{$\begin{array}{l}\text { Parámetros } \\
\text { generales }\end{array}$} & Área & $6.82 \mathrm{~km}^{2}$ \\
\hline & Perímetro & $14.7 \mathrm{~km}$ \\
\hline & Longitud del cauce principal & $3.939 \mathrm{~km}$ \\
\hline \multirow{6}{*}{$\begin{array}{l}\text { Parámetros de } \\
\text { forma }\end{array}$} & Coeficiente de compacidad & 1.89 \\
\hline & Factor de forma & 0.44 \\
\hline & Razón de elongación & 0.20 \\
\hline & Índice de circularidad & 0.39 \\
\hline & $\begin{array}{l}\text { Longitud del lado mayor del } \\
\text { rectángulo equivalente }\end{array}$ & $7.89 \mathrm{~km}$ \\
\hline & $\begin{array}{l}\text { Longitud del lado menor del } \\
\text { rectángulo equivalente }\end{array}$ & 0.87 km \\
\hline
\end{tabular}




\begin{tabular}{|c|c|c|}
\hline \multirow{8}{*}{$\begin{array}{l}\text { Parámetros de } \\
\text { relieve }\end{array}$} & Desnivel altitudinal & $996 \mathrm{~m}$ \\
\hline & Cota mínima & $597 \mathrm{msnm}$ \\
\hline & Cota máxima & $1593 \mathrm{msnm}$ \\
\hline & Elevación media & $1144 \mathrm{mnsm}$ \\
\hline & Coordenada del centroide $X$ & 547084 \\
\hline & Coordenada del centroide $Y$ & 8758966 \\
\hline & $\begin{array}{c}\text { Pendiente de la cuenca (método } \\
\text { de Alvord) }\end{array}$ & $42 \%$ \\
\hline & Pendiente del cauce principal & $22 \%$ \\
\hline
\end{tabular}

\section{Precipitación}

Análisis de consistencia de precipitación. En la Figura 4 se observa la curva de doble masa de cada grupo, donde se pueden visualizar las gráficas entre la precipitación acumulada y la precipitación promedio acumulada de las estaciones según el grupo formado. Del análisis se observa que no existen saltos significativos y que las líneas generadas de las estaciones forman rectas invariables; esto confirma que las estaciones tienen registros homogéneos y son consistentes. 
Tecnología y

\section{Ciencias $₫$ Agua}

2022, Instituto Mexicano de Tecnología del Agua

Open Access bajo la licencia CC BY-NC-SA 4.0

(https://creativecommons.org/licenses/by-nc-sa/4.0/)



Análisis de Doble Masa



- Ríchanaki - Satipo - Puetto Oxopa - Oxapampa
Análisis de Doble Masa

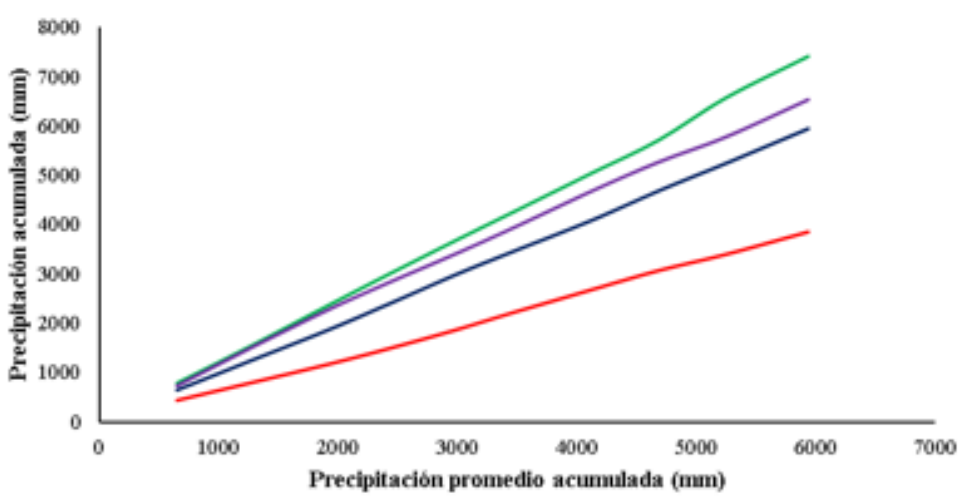

- Tarma - Huasahuasi - Ricran - La oroya

Análisis de Doble Masa

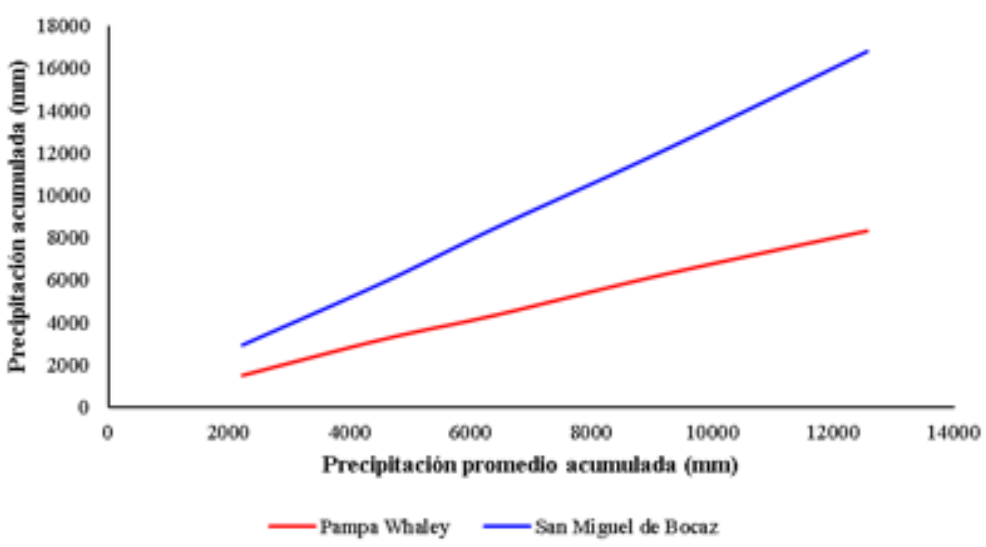

Figura 4. Análisis de doble masa.

Precipitación de la microcuenca. Mediante el método de las isoyetas se realizaron 12 distribuciones (enero a diciembre) cada $10 \mathrm{~mm}$ para la precipitación promedio mensual y cada $50 \mathrm{~mm}$ para la precipitación anual (Figura 5) de la cuenca del río Perené mediante el software ArcGis, para determinar la precipitación en la cuenca de estudio (Cashingari). 
2022, Instituto Mexicano de Tecnología del Agua

Tecnología y

Open Access bajo la licencia CC BY-NC-SA 4.0

(https://creativecommons.org/licenses/by-nc-sa/4.0/)

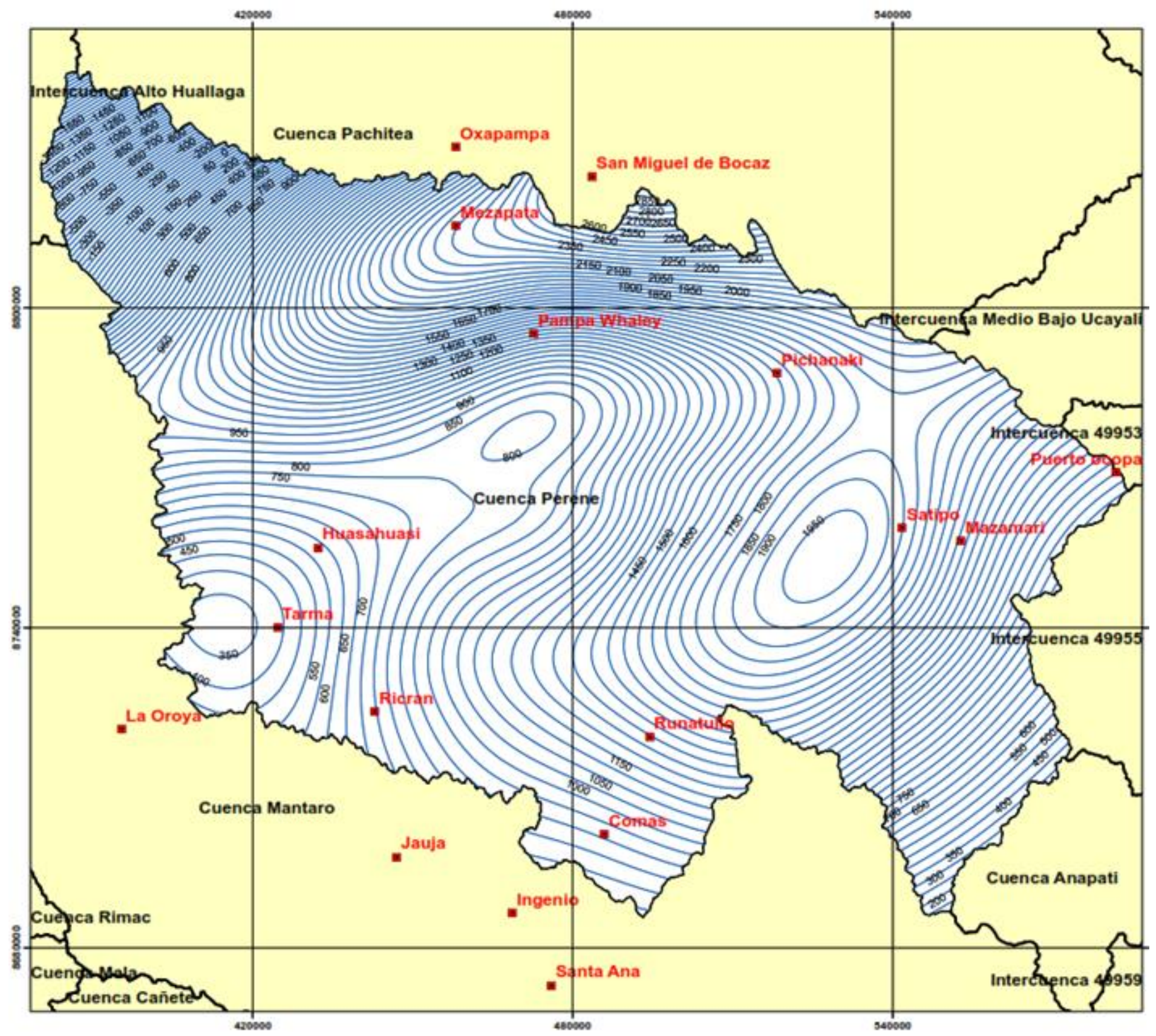

Figura 5. Mapa de isoyetas de la cuenca Perené para determinar la precipitación anual. 
Tecnología y

Ciencias $₫$ Agua
2022, Instituto Mexicano de Tecnología del Agua

Open Access bajo la licencia CCBY-NC-SA 4.0

(https://creativecommons.org/licenses/by-nc-sa/4.0/)

En la Figura 6 se muestra la precipitación mensual promedio de la microcuenca, donde el mes de mayor precipitación es diciembre, con $252.6 \mathrm{~mm}$, mientras que el mes de junio $(51.4 \mathrm{~mm})$ es el más seco, con la menor precipitación.

\section{Precipitación promedio mensual}



Figura 6. Precipitación promedio mensual de la cuenca.

\section{Caudal promedio}


Características geomorfológicas. La cuenca elegida para el método de transposición de caudales es la cuenca Ourohuari. Las características geomorfológicas e hidrometeorológicas entre ambas cuencas son similares y no existen variaciones significativas. Para verificar estas similitudes, se realizaron los respectivos análisis (Tabla 7).

Tabla 7. Comparación de las características geomorfológicas de las microcuencas Cashingari y Ourohuari.

\begin{tabular}{|c|c|c|c|}
\hline \multirow{4}{*}{$\begin{array}{c}\text { Tipo de } \\
\text { parámetro }\end{array}$} & Parámetro & \multicolumn{2}{|c|}{ Cuenca } \\
\cline { 2 - 4 } & & Cashingari & Ourohuari \\
\hline \multirow{4}{*}{$\begin{array}{c}\text { Parámetros } \\
\text { generales }\end{array}$} & Perímetro & $6.82 \mathrm{~km}^{2}$ & $8.1 \mathrm{~km}^{2}$ \\
\cline { 2 - 4 } & Desnivel altitudinal & $996 \mathrm{~m}$ & $745 \mathrm{~m}$ \\
\cline { 2 - 4 } & $\begin{array}{c}\text { Longitud del cauce } \\
\text { principal }\end{array}$ & $3.939 \mathrm{~km}$ & $4.5 \mathrm{~km}$ \\
\hline \multirow{4}{*}{$\begin{array}{c}\text { Parámetros } \\
\text { de forma }\end{array}$} & $\begin{array}{c}\text { Coeficiente de } \\
\text { compacidad }\end{array}$ & 1.89 & 1.33 \\
\cline { 2 - 4 } & Densidad de drenaje & 0.73 & 0.56 \\
\cline { 2 - 4 } & Factor de forma & 0.44 & 0.40 \\
\cline { 2 - 4 } & Razón de elongación & 0.20 & 0.24 \\
\cline { 2 - 4 } & Indice de circularidad & 0.39 & 0.57 \\
\hline & Cota mínima & $597 \mathrm{msnm}$ & $675 \mathrm{msnm}$ \\
\hline
\end{tabular}


2022, Instituto Mexicano de Tecnología del Agua

Ciencias $\stackrel{\Xi}{\Im}$ Agua

Open Access bajo la licencia CC BY-NC-SA 4.0

(https://creativecommons.org/licenses/by-nc-sa/4.0/)

\begin{tabular}{|c|c|c|c|}
\hline \multirow{1}{*}{$\begin{array}{c}\text { Parámetros } \\
\text { de relieve }\end{array}$} & \begin{tabular}{c} 
Cota máxima \\
Elevación media \\
\cline { 2 - 4 }
\end{tabular} & $1593 \mathrm{msnm}$ & $\begin{array}{c}1420 \\
\mathrm{msnm}\end{array}$ \\
\cline { 2 - 4 } & $\begin{array}{c}\text { Longitud del lado mayor } \\
\text { engitudente } \\
\text { del rectángulo } \\
\text { equivalente }\end{array}$ & $7.89 \mathrm{~km}$ & $4.90 \mathrm{~km}$ \\
\cline { 2 - 4 } & $\begin{array}{c}\text { Pendiente de la cuenca } \\
\text { (método de Alvord) }\end{array}$ & $42 \%$ & $27 \%$ \\
\hline
\end{tabular}

La proporción del área entre ambas cuencas está dentro del rango de 0.5-1.5; la diferencia entre la longitud de sus cauces principales es mínima, además, todos los parámetros de forma son similares, a excepción del coeficiente de compacidad, lo que demuestra que la cuenca Cashingari presenta una forma más alargada y la cuenca Ourohuari tiene una forma más redondeada, por ende, las características geomorfológicas entre las cuencas mencionadas son similares.

Características hidrometeorológicas. Los valores de precipitación promedio mensual de ambas cuencas se muestran en la Tabla 8, y el gráfico de la variación de precipitación total mensual se muestra en la Figura 6. 
Tecnología y

Ciencias $₫$ Agua
2022, Instituto Mexicano de Tecnología del Agua

Open Access bajo la licencia CC BY-NC-SA 4.0

(https://creativecommons.org/licenses/by-nc-sa/4.0/)

Tabla 8. Precipitación promedio mensual de las cuencas Cashingari y Ourohuari.

\begin{tabular}{|l|c|c|}
\hline \multirow{2}{*}{ Meses } & \multicolumn{2}{|c|}{ Precipitación (mm) } \\
\cline { 2 - 3 } & Cashingari & Ourohuari \\
\hline Enero & 228.04 & 261.9 \\
\hline Febrero & 223.1 & 196.7 \\
\hline Marzo & 209.9 & 125.2 \\
\hline Abril & 129.9 & 82.5 \\
\hline Mayo & 66.8 & 62.9 \\
\hline Junio & 51.4 & 82.6 \\
\hline Julio & 60.3 & 76.4 \\
\hline Agosto & 70.7 & 92.0 \\
\hline Setiembre & 100.1 & 190.7 \\
\hline Octubre & 156.6 & 173.5 \\
\hline Noviembre & 173.0 & 263.4 \\
\hline Diciembre & 252.6 & $\mathbf{1 8 6 0 . 0}$ \\
\hline Anual & $\mathbf{1 7 2 3 . 0 9}$ & \\
\hline
\end{tabular}

La Figura 7 muestra el comportamiento cinético de las precipitaciones del promedio mensual de las cuencas de Cashingari y Ourohuari, donde se observa para ambas cuencas un comportamiento similar; los meses de mayo a septiembre tuvieron menos de $100 \mathrm{~mm}$ de 
precipitaciones; en enero, febrero y diciembre, las precipitaciones fueron de unos $250 \mathrm{~mm}$.

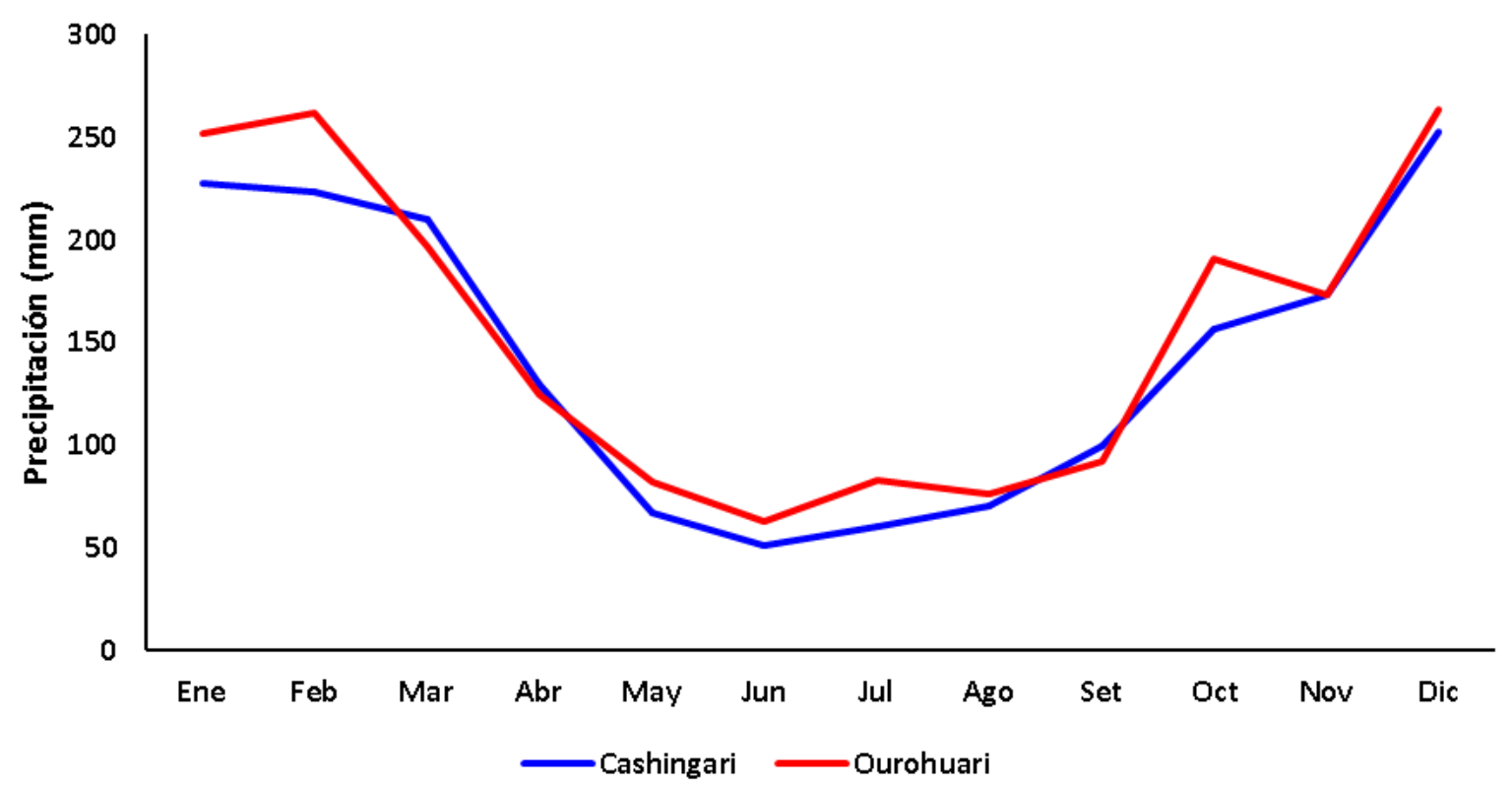

Figura 7. Variación de la precipitación promedio mensual entre la cuenca Cashingari y Ourohuari.

Se realizó una prueba $t$-student para muestras independientes, con el fin de comprobar si la precipitación promedio mensual (Tabla 9) entre las cuencas son relativamente similares. La cuenca Cashingari y la cuenca Ourohuari mostraron estadísticamente tener una distribución normal, con $P$-value de 0.193 y 0.071 , respectivamente, a un nivel de confianza del $95 \%$. 
Tabla 9. Prueba $t$-student para muestras independientes.

\begin{tabular}{|c|c|c|c|c|c|}
\hline \multirow{2}{*}{$\begin{array}{c}\text { Grupos (G) } \\
\text { precipitación } \\
(\mathbf{m m})\end{array}$} & \multicolumn{4}{|c|}{ t-test variables independientes } \\
\cline { 2 - 6 } & Media G1 & Media G2 & t-value & df & p-value \\
\hline $\begin{array}{c}\text { Cashingari } \\
\text { vs. } \\
\text { Ourohuari }\end{array}$ & 143.53 & 154.99 & -0.371 & 22 & 0.7137 \\
\hline
\end{tabular}

En la Tabla 9 se observa que con un $p$-value de 0.7137 superior al 0.05 trabajando con un nivel de confianza del $95 \%$, la precipitación entre la cuenca Cashingari y Ourohuari mostraron estadísticamente no significativo o similares con relación a la precipitación con un total de 22 de grados de libertad, considerando una muestra de 12 por cada cuenca. La Figura 8 muestra los diagramas de cajas que indican una similitud de las medias de precipitaciones de ambas cuencas en un periodo de 12 meses. 


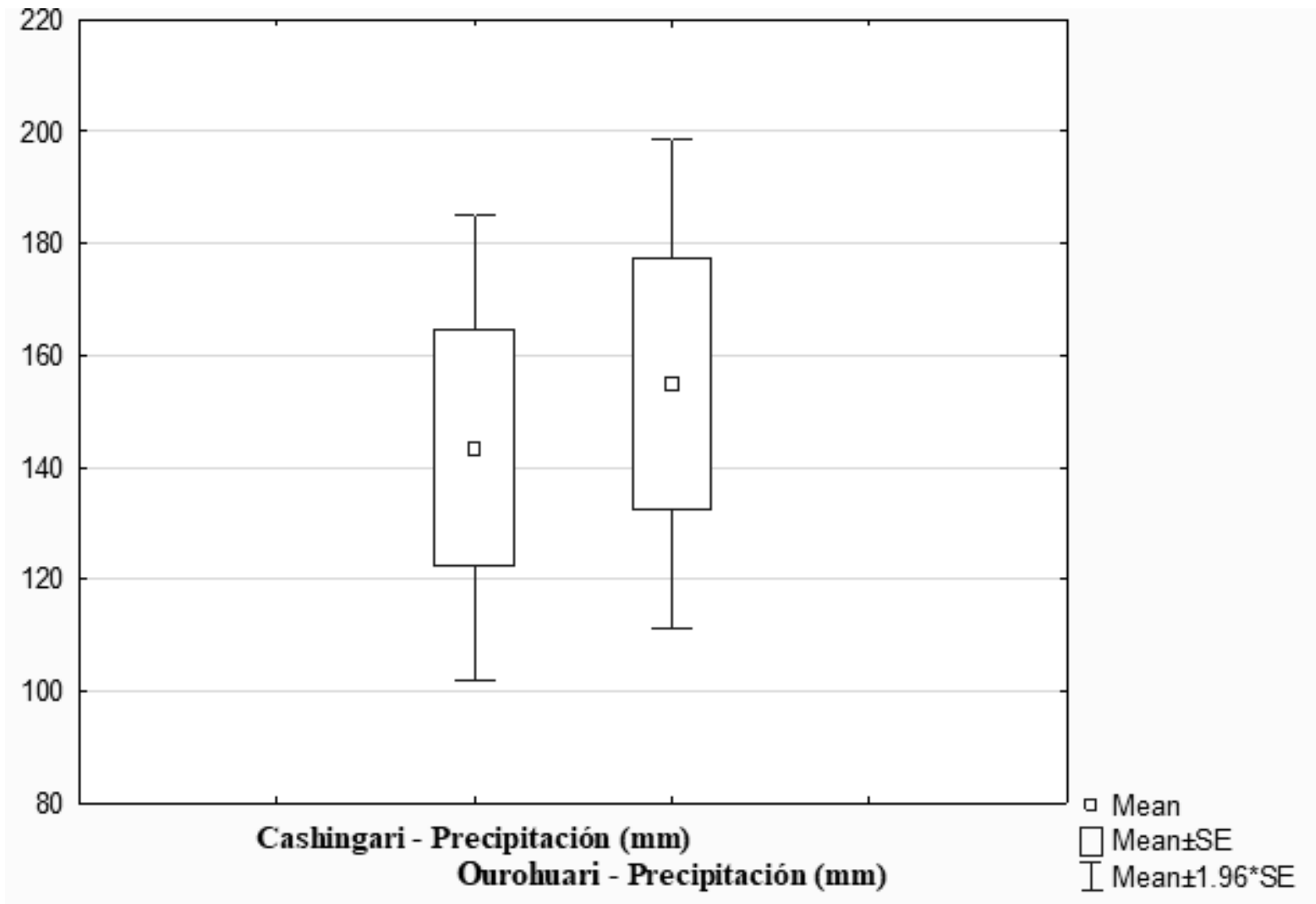

Figura 8. Diagrama de cajas de precipitación mensual entre la cuenca Cashingari y Ourohuari.

Zonas de vida. Dentro de la cuenca Cashingari se identificaron dos zonas de vida (Figura 9) que explican el comportamiento hidrológico: bosque húmedo premontano tropical (bh - PT) y bosque húmedo tropical (bh - T). Asimismo, la cuenca Ourohuari (Figura 10) presenta las mismas zonas de vida que la cuenca Cashingari, la cual indicó una similitud de los parámetros climáticos, principalmente de la temperatura y precipitación entre ambas cuencas. 
Tecnología y

\section{Ciencias $₫$ Agua}

2022, Instituto Mexicano de Tecnología del Agua

Open Access bajo la licencia CC BY-NC-SA 4.0

(https://creativecommons.org/licenses/by-nc-sa/4.0/)

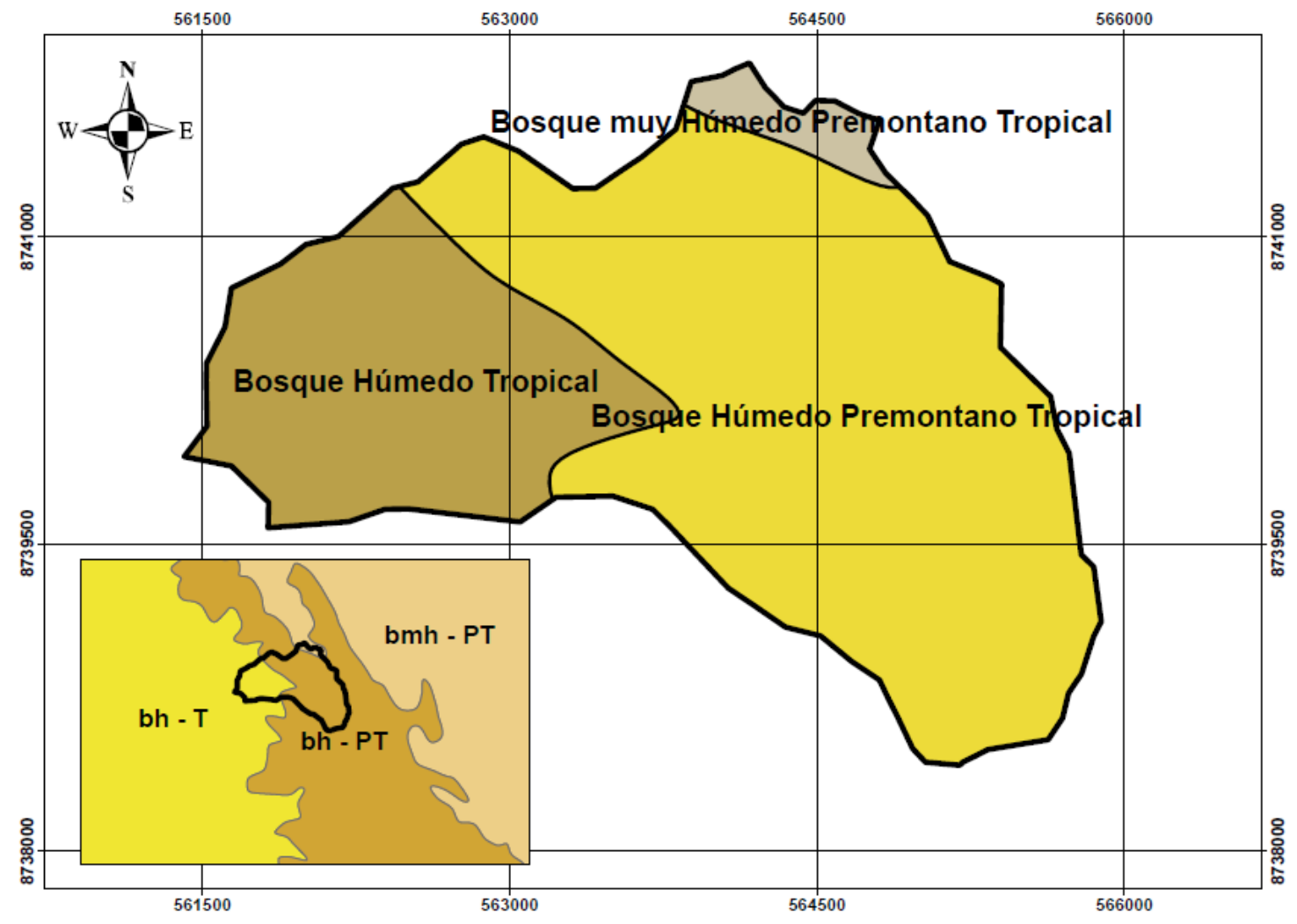

Figura 9. Zonas de vida de la cuenca Cashingari. 
Teçnología y

Ciencias $₫$ Agua
2022, Instituto Mexicano de Tecnología del Agua

Open Access bajo la licencia CC BY-NC-SA 4.0

(https://creativecommons.org/licenses/by-nc-sa/4.0/)

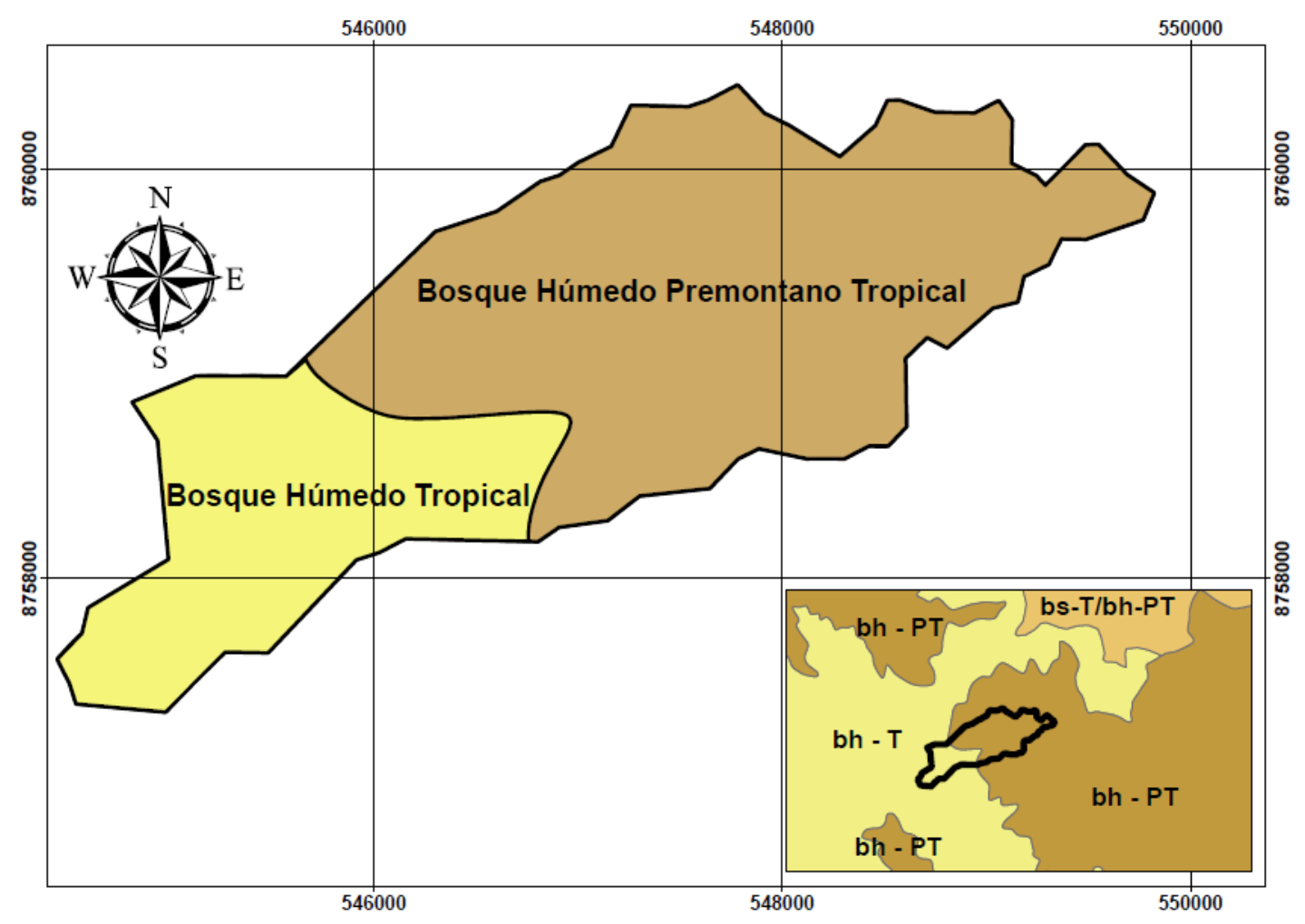

Figura 10. Zonas de vida de la cuenca Ourohuari.

Debido a que estadísticamente la precipitación entre ambas cuencas es similar, al igual que las características climatológicas, por presentar las mismas zonas de vida y que, finalmente, las características geomorfológicas no difieren entre cuencas, se estimaron los caudales para la microcuenca Cashingari, como se muestra en la Figura 11. Se observa que el caudal máximo mensual promedio es de $329.3 \mathrm{l} / \mathrm{s}$, correspondiente al mes de marzo; mientras que el caudal mínimo mensual promedio es de $57.0 \mathrm{l} / \mathrm{s}$ en el mes de agosto. De igual forma, de los caudales estimados, se puede deducir que el río presenta una corriente de agua regular durante todo el año. 


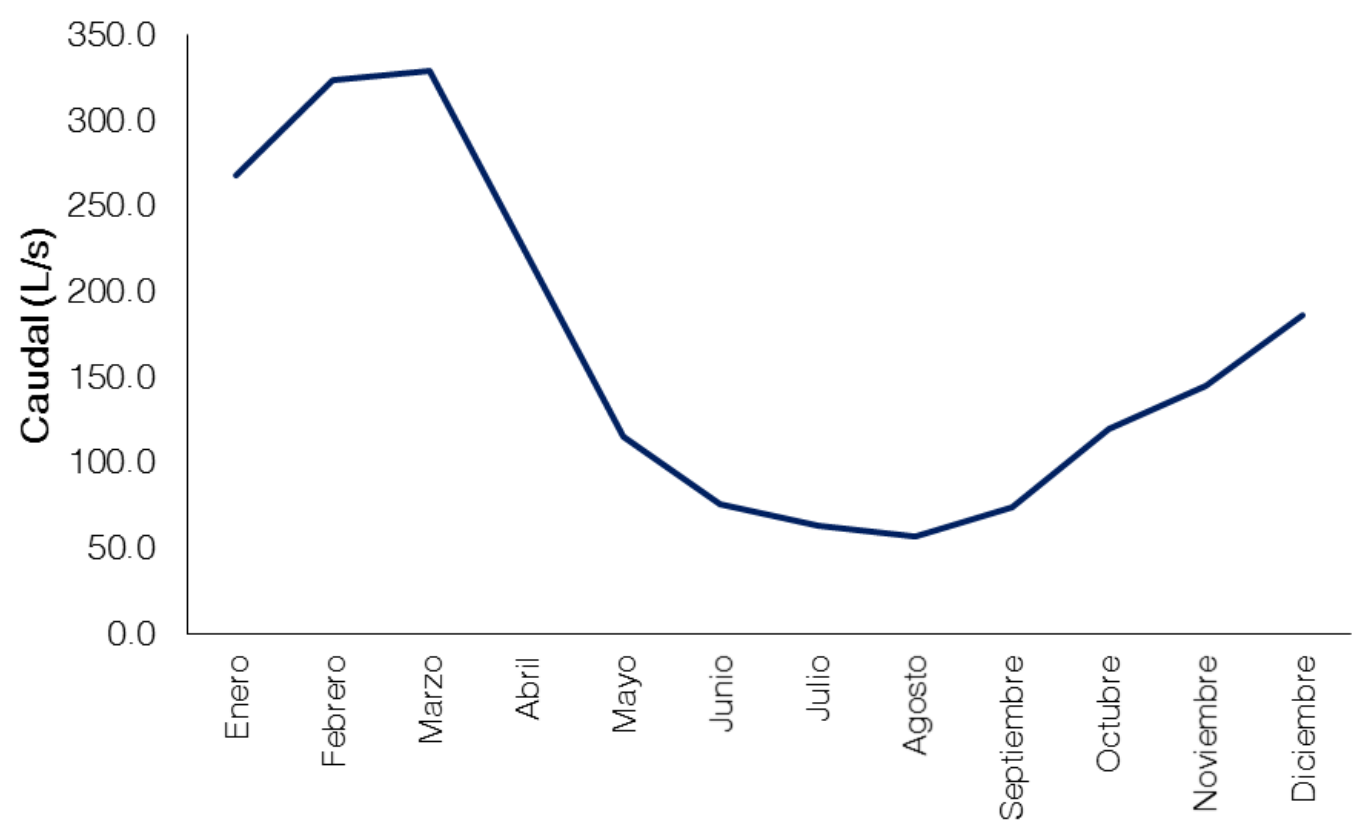

—Caudal Promedio

Figura 11. Caudales promedio mensual estimados de la cuenca Cashingari.

\section{Máximas avenidas}

Caudal de máximas avenidas. La microcuenca Cashingari se encuentra dentro de la región 7 , según el mapa de regionalización de avenidas del Perú (Trau \& Gutierrez, 1979), por ende, los coeficientes que le 
Teçnología y

Ciencias $₫$ Agua
2022, Instituto Mexicano de Tecnología del Agua

Open Access bajo la licencia CC BY-NC-SA 4.0

(https://creativecommons.org/licenses/by-nc-sa/4.0/)

corresponden son: $C 1=0.22, C 2=0.37, m=0.24, n=0.04$. Los periodos de retorno elegidos son 5, 10, 25, 50 y 100 años. Los caudales de avenidas se muestran en la Tabla 10 y Figura 12.

Tabla 10. Caudales de avenidas estimados con la envolvente regional de Creager de la microcuenca Cashingari.

\begin{tabular}{|c|c|}
\hline Periodo de retorno (años) & Caudal de avenida $\left.\mathbf{~ ( m}^{\mathbf{3}} \mathbf{/ s}\right)$ \\
\hline 5.0 & 5.4 \\
\hline 10.0 & 7.7 \\
\hline 25.0 & 10.8 \\
\hline 50.0 & 13.1 \\
\hline 100.0 & 15.4 \\
\hline
\end{tabular}




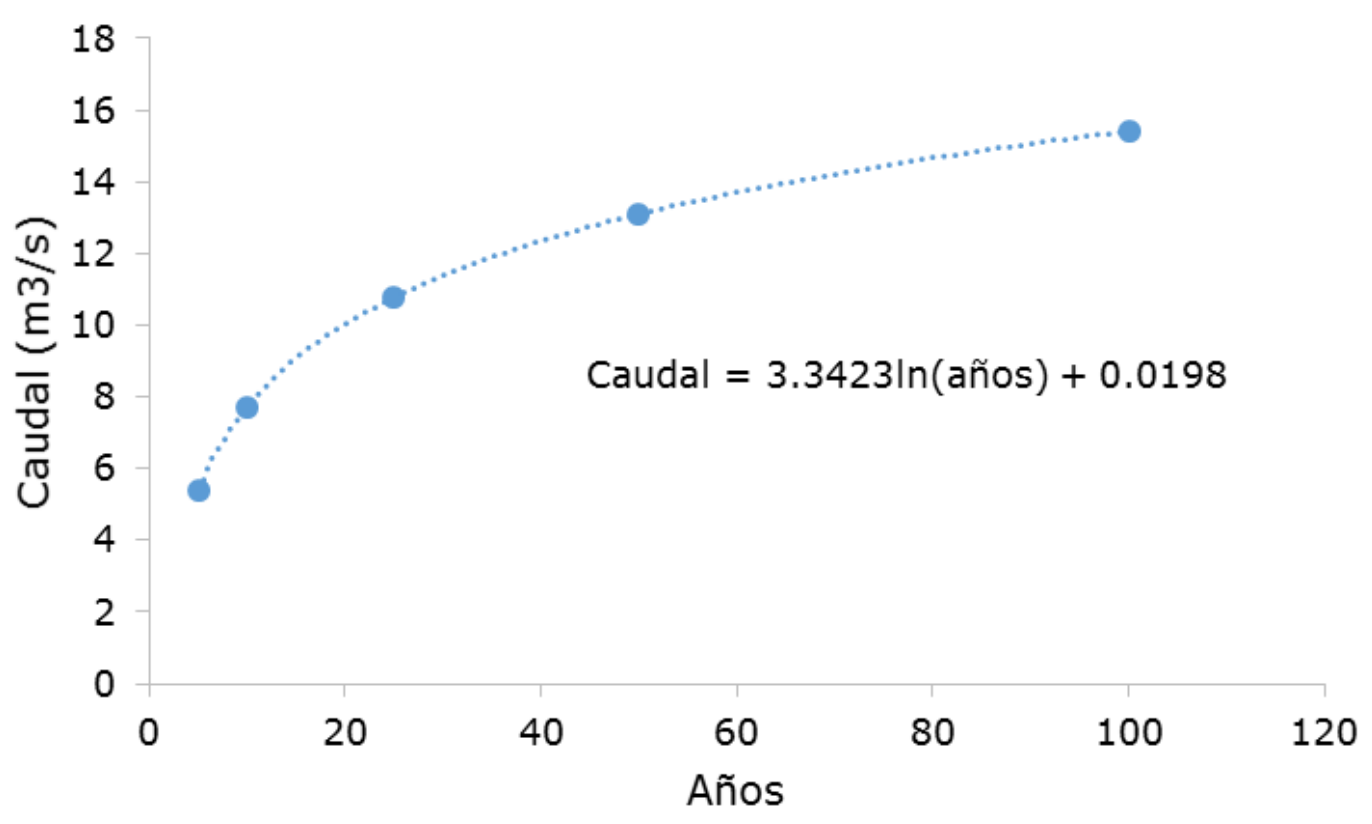

Figura 12. Regresión logarítmica de estimación de caudal de Cashingari en función al período de retorno.

\section{Conclusiones}

Geomorfológicamente, la cuenca presenta una forma alargada ; asimismo, mediante el método de Alvord, la pendiente de la cuenca es del $42 \%$; el de su cauce principal es de $22 \%$, y el desnivel altitudinal es de 996 m; se clasifica como empinada. Por tanto, la topografía de la cuenca favorece la 
generación de electricidad a través de una pico central debido a una mayor energía potencial en la caída del agua.

Estadísticamente, la precipitación entre la cuenca de estudio y la cuenca de donde se transportaron los caudales son similares; además, las zonas de vida de ambas cuencas son las mismas, lo que indican una similitud en los parámetros climatológicos.

Los caudales estimados para la microcuenca Cashingari son regulares durante todo el año, incluso en los meses secos con baja precipitación $(57.0 \mathrm{~mm})$. Esto favorece en gran manera el desarrollo de una pico central con niveles de generación inferior a los $5 \mathrm{~kW}$ debido a la disponibilidad del recurso hídrico de manera regular e ininterrumpida durante los 12 meses del año, no viéndose afectada la generación de energía por falta de agua; además, para este tipo de central, no se requiere de grandes caudales de generación.

Los resultados del estudio hidrológico de la microcuenca Cashingari impulsarán la actual iniciativa de generación de hidroenergía en esta zona de la selva del Perú (Satipo), considerando la topografía favorable y la gran disponibilidad del recurso hídrico, las cuales son dos parámetros de entrada fundamentales para el desarrollo de generación de electricidad mediante la caída del agua (caudal y carga hidráulica).

\section{Referencias}

Bilewu, S., Sule, B., \& Ayanshola, A. (2015). Optimum parameter selection for the morphometric description of watersheds: A case 
study of central Nigeria. Journal of Ecological Engineering, 16(4), 29-35. Recuperado de https://doi.org/10.12911/22998993/59344

Chouhan, K., Kisheorey, G., \& Shah, M. (2017). Modelling, fabrication \& analysis of Pelton turbine for different head and materials. International Journal of Computational Engineering Research, 7(02), 2250-3005. Recuperado de www.ijceronline.com

De-Lima, G., Marcellini, S. S., Neill, C. R., \& Salla, M. R. (2017). Preliminary estimate of floods discharge in Brazil using Creager envelope curves. Brazilian Journal of Water Resources, 22(46), 6. Recuperado de https://doi.org/10.1590/2318-0331.011716078

Edeoja, A., Ibrahim, S., \& Kucha, E. (2015). Suitability of Picohydropower technology for addressing the Nigerian energy crisis- $A$ review. International Journal of Engineering Inventions, 4(9), 1740. Recuperado de www.ijeijournal.com

Ezemonye, M., \& Emeribe, C. (2013). Appraisal of the hydrological potential of Ungauged basin using morphometric parameters. Ethiopian Journal of Environmental Studies and Management, 6(4), 376-380. Recuperado de https://doi.org/10.4314/ejesm.v6i4.5

Fatoyinbo, B., Stretch, D., Amoo, O., \& Allopi, D. (2017). Catchment yield prediction in an Ungauged basin using PyTOPKAPI. International Scholarly and Scientific Research \& Innovation, 11(3), 281-288. Recuperado de https://waset.org/publications/10006803/catchment-yieldprediction-in-an-ungauged-basin-using-pytopkapi 
Fry, L., Hunter, T., Phanikumar, M., Fortin, V., \& Gronewold, A. (2013). Identifying stream gage networks for maximizing the effectiveness of regional water balance modeling. Water Resources Research,49, 2689-2700. Recuperado de https://doi.org/10.1002/wrcr.20233

Fujii, M., Tanabe, S., Yamada, M., Mishima, T., Sawadate, T., \& Ohsawa, S. (2017). Assessment of the potential for developing mini/micro hydropower: A case study in Beppu City, Japan. Journal of Hydrology: Regional Studies, 11, 107-116. Recuperado de https://doi.org/10.1016/J.EJRH.2015.10.007

Gavit, B., Purohit, R., Singh, P., Kothari, M., \& Jain, H. (2016). Grouping of geomorphic parameters in selected watershed using principal component analysis for hydrological modelling. International Research Journal of Environment Sciences, 5(12), 7-13. Recuperado de http://www.isca.in/IJENS/Archive/v5/i12/2.ISCAIRJEvS-2016-118.pdf

Hortness, J. (2006). Estimating low-flow frequency statistics for unregulated streams in Idaho (Report 2006-5035). Denver, USA: U.S. Department of the Interior, U.S. Geological Survey.

Jimenez, B., \& Galizia, J. (2012). Diagnóstico del agua en la Américas. México: Foro Consultivo Científico y Tecnológico. Recuperado de http://www.ianas.org/water/book/peru.pdf

Kusre, B. C., Baruah, D. C., Bordoloi, P. K., \& Patra, S. C. (2010). Assessment of hydropower potential using GIS and hydrological modeling technique in Kopili River basin in Assam (India). Applied 
Tecnología y

Ciencias $₫$ Agua
2022, Instituto Mexicano de Tecnología del Agua

Open Access bajo la licencia CC BY-NC-SA 4.0

(https://creativecommons.org/licenses/by-nc-sa/4.0/)

Energy,

$87(1)$,

298-309.

Recuperado

de https://doi.org/10.1016/J.APENERGY.2009.07.019

Meier, P., Zolezzi, E., Bogach, S., Muir, T., \& Bazex, K. (2011). Peru opportunities and challenges of small hydropower development. Washington, DC, USA. Recuperado de https://openknowledge.worldbank.org/bitstream/handle/10986/17 500/636680W POPeru000Box0361524B0PUBLIC0.pdf

Negrel, J., Kosuth, P., \& Bercher, N. (2011). Estimating river discharge from earth observation measurements of river surface hydraulic variables. Hydrology and Earth System Sciences, 15, 2049-2058. Recuperado de https://doi.org/10.5194/hess-15-2049-2011

Reyes, A., Barroso, F., \& Carvajal, Y. (2010). Guía básica para la caracterización morfométrica de cuencas hidrográficas. Santiago de Cali, Colombia: Universidad del Valle.

Sidek, L., Zaki, A., Mustaffa, Z., Ibrahim, M., Muda, Z., Thiruchelvam, S., \& Basri, H. (2013). Hydrological assessment for mini hydropower potential at Sungai Pahang Temerloh. In: 4th International Conference on Energy and Environment (pp. 1-4). Selangor, Malasia: IOP Conf. Series: Earth and Environmental Science. Recuperado de https://doi.org/doi:10.1088/1755$1315 / 16 / 1 / 012046$

Soulis, K., Manolakos, D., Anagnostopoulos, J., \& Papantonis, D. (2016). Development of a geo-information system embedding a spatially distributed hydrological model for the preliminary assessment of the hydropower potential of historical hydro sites in poorly gauged 
areas. Renewable Energy, 92, 222-232. Recuperado de https://doi.org/10.1016/J.RENENE.2016.02.013

Trau, W., \& Gutierrez, R. (1979). Análisis regional de las avenidas en los ríos del Perú. ONERN. Lima, Perú: ONERN. Recuperado de http://repositorio.ana.gob.pe/handle/ANA/1066

Uniyal, V., Kanojia, N., \& Pandey, K. (2016). Design of 5kw pico hydro power plant using Turgo turbine. International Journal of Scientific \& Engineering Research, 7(12), 363-367. Recuperado de http://www.ijser.org

World Bank. (2017). Rural electrification project. Washington, DC, USA: World Bank. Recuperado de www.worldbank.org 